\title{
Computer Simulation of Electroosmotic Soils Treatment
}

\author{
Maxim Gucal $^{1 *}$, Andrey Pokatilov ${ }^{1}$ \\ ${ }^{1}$ T.F. Gorbachev Kuzbass State Technical University, Mining Institute, 650000, Kemerovo, Russia
}

\begin{abstract}
The technique of experimental and analytical assessment of electrical and hydrodynamic parameters of clay soils as a result of physical and computer modelled processes in electroosmotic treatment zone is presented. The algorithm is given for calculation of the coefficients of hydraulic permeability, electroosmotic permeability and electroosmotic activity comprising the determination of geometric boundaries of the zones subjected to electric treatment, the initial filtration rate, and the calculation of the electric field and pressure. Spatial and temporal projections of dependent parameters at different stages of electric treatment are obtained.
\end{abstract}

\section{Introduction}

Electroosmotic treatment is one of the most effective ways to change the properties of water-saturated low permeability clay soils. Associated electroosmotic treatment processes are such as electrolysis, various exchange reaction, processes of formation and accumulation of new chemical compounds [1-4]. The classical equation of electroosmotic filtering is as follows [5]:

$$
\bar{V}=k_{e} \overline{\operatorname{grad}} \varphi+k_{f} \overline{\operatorname{grad}} h=k_{f} \overline{\operatorname{grad}}\left(k_{a} \varphi+h\right)
$$

where $\bar{V}$ - velocity of particles (ions); $\varphi$ - electric potential; $h$ - water head; $, k_{f}, k_{e}, k_{a}-$ coefficients of hydraulic permeability, electroosmotic permeability and electroosmotic activity, respectively.

Taking into account the non-stationary physical processes, heterogeneity and anisotropy of the properties of the soil in the area of electric treatment, the analytic solution of equation (1) with a fairly acceptable accuracy is not possible. The parameters characterizing the process of electroosmotic treatment of soil include a set of parameters $\bar{V}, \varphi, h k_{f}, k_{e}, k_{a}$ from the equation (1). According to the standards, for the experimental determination of the filtration characteristics it is necessary to have special laboratory equipment described in a number of papers [6-13]. In particular, with the equipment described in these works it is possible using soil samples to determine in the laboratory the dependences of the parameters $k_{f}, k_{e}, k_{a}$ on the porosity, the soil moisture, an electric field intensity, the processing

\footnotetext{
* Corresponding author: gmv.tgm @,kuzstu.ru
} 
time and the consumption of electrical current, and relevant migrations of liquids filling the pores in the inter-electrode space.

Often with the purpose of detailing the data, researchers resort to mathematical modeling of various stages of physical experiments $[9,14,15]$. In particular, the computer modeling is one of the most effective methods for the study of complex systems. To explore computer models is easier and more convenient than to run live experiments, as the latter can be very labor- and resource-intensive and may give unpredictable results. Computer models help us to identify the main factors that determine the properties of the object under study or process, to forecast the impact of the results from the taken decisions, see the problem in its development.

For a long time, the researchers of the Kuzbass State Technical University have been conducting investigations related to geophysical monitoring of processes in the areas of deliberate impact on the physical and mechanical properties of rocks $[9,14]$, wherein the specific electrical resistance (SER) of soil is the main informing parameter. They used both the integral measurement circuits (electrical sounding and profiling, georadar) and the differentiated ones to determine the true SER (downhole electric and induction logging, microsensors). In the interpretation of geophysical data, the main difficulty lies in the unsteadiness of the processes under review: during processing of the solid mass its electroand hydrodynamic parameters are continuously changing. To enhance the monitored information content, it is advisable to use an approach similar to the method of successive change of stationary states: the physical processes in the processing zone to be considered as stationary in the intervals between the geophysical measurements, and changes in the massif properties to be evaluated by the data of discrete measurements.

In this paper, we consider the indirect assessment of the above-mentioned electric and hydrodynamic parameters using the results of physical and computer modeling of the area of electroosmotic treatment of the clay soil.

The block-diagram of experimental computer model of electroosmotic filtration is shown in Fig. 1. The physical basis of the proposed method is the equation of electroosmotic fluid displacement velocity in the filtration channel, subordinated to the theory of Helmholtz-Smoluchowski [11, 12, 14, 16, 17]:

$$
\bar{V}=-\frac{\varepsilon \varsigma}{\mu} \operatorname{grad} \phi
$$

where $\varepsilon$ - absolute dielectric constant, $\mathrm{F} / \mathrm{m} ; \mu$ - the coefficient of dynamic viscosity of the solution, $\mathrm{Pa} \cdot \mathrm{s} ; \varsigma$ - zeta potential, V.

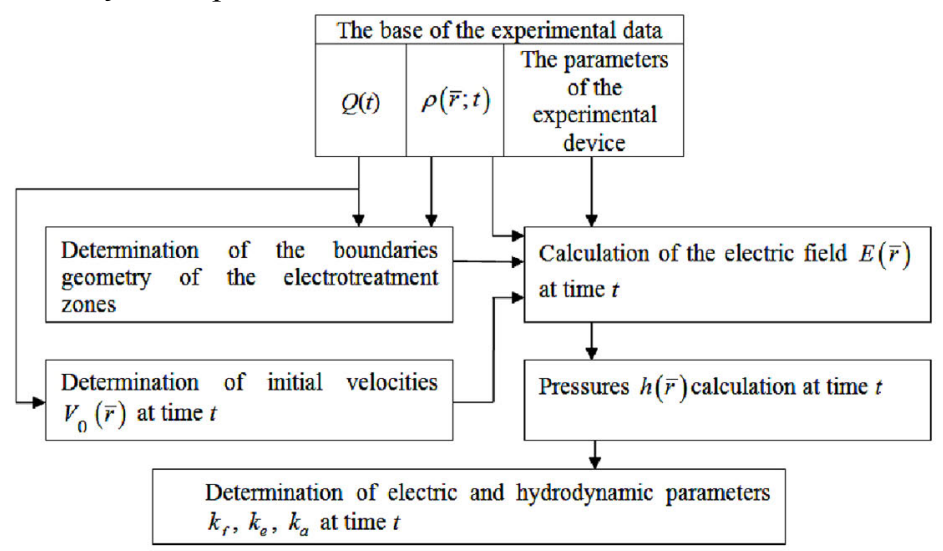

Fig. 1. Block-diagram of experimental computer model: $Q$ - flow; $\rho$ - SER; $\bar{r}$ - coordinate; I-V calculation stages 
When filtering in a porous medium, the module of effective velocity $V_{e f}$ (flow) is produced on the basis of dimensional analysis:

$$
V_{e f}=C \sigma_{0} \frac{m^{2} R}{\mu} E=\frac{m \varsigma \varepsilon}{\mu} E=k_{e} E
$$

where $C$ - constant; $\sigma_{0}$ - specific volume density of diffuse layer ions charge, $\mathrm{C} / \mathrm{m}^{3} ; t$ - soil porosity; $R$ - hydraulic pore radius, $\mathrm{m} ; E$ - electric field intensity, $\mathrm{V} / \mathrm{m}$.

The calculation is divided into five stages $(\mathrm{I}-\mathrm{V})$.

At the first stage, based on analysis of changes in the relative values of SER $\rho(\bar{r})$, the sizes of characteristic zones formed during soil treatment are determined.

The second stage is responsible for the calculation of the initial electroosmosis velocity taken as a reference. The liquid output $\mathrm{Q}$ from the electrode-injector recorded in the course of the experiment serves as the data for calculation.

At the third stage, the calculation of the velocity of the electroosmosis process change is made, taking the difference of the areas under the graphs SER $\rho(\bar{r})$ as the characteristic of the process change. The calculation is made using programs of PDE MATLAB library section that implement the finite element method. The task is reduced to the solution of the Laplace equation with the parameters of the massif corresponding to the real conditions:

$$
\Delta \varphi(x, z)=0
$$

where $x, z$ - horizontal and vertical coordinates.

The conditions at infinity and at the interface are taken as traditional:

$$
\begin{aligned}
& \left.\varphi_{1}\right|_{x^{2}+z^{2} \rightarrow \infty} \rightarrow 0 ;\left.\quad \varphi_{i}\right|_{x=x_{i}}=\left.\varphi_{i+1}\right|_{x=x_{i}} ;\left.\frac{\partial \varphi}{\partial z}\right|_{z=z_{0}}=0 ; \\
& \left.\frac{\partial \varphi_{i}}{\partial x}\right|_{x=x_{i}}=\left.\frac{\partial \varphi_{i+1}}{\partial x}\right|_{x=x_{i}}
\end{aligned}
$$

Calculation of pressures (phase IV) is based on the electric- hydrodynamic analogy, previously established for the progressive and flat-radial flow of filtered fluid. The dependence of the potential function of the electric field on the pressure function can be expressed as follows:

$$
\varphi(\bar{r})=K_{2}\left[K_{3}-h(\bar{r})\right]
$$

where $\varphi(\bar{r})$ - the function determined by integrating function $E(\bar{r}) ; K_{2}$ - the constant depending on the electrochemical, dielectric, conductive properties and porosity of the environment; $K_{3}$ - the constant depending on the limit value of pressure. Determination of a group of electric- and hydrodynamic parameters in the settlement times (Phase V) is made by solving the equations (1) - (6) with the substitution of intermediate results.

\section{Results and discussion}

Fig. 2 and 3 show initial data for calculations of stages I and II as experimental plots obtained on a 3-D physical model. Fig. 2 shows plots of the relative SER $\rho(x, I \times t)$, where $x$ coordinate on the axis of the apparatus, $I$ - current, $t$ - time, taken as the initial basis of experimental data, and the boundaries of the main zones of electroosmosis (drainage and accumulation of moisture). Fig. 3 shows the data of the fluid output $Q$ from electrode-injector, which served as the baseline for the calculation of the reference filtration velocity. 
Fig. 4 shows the results of calculation of the relative electroosmosis velocity $V / V_{0}(t, x)$.

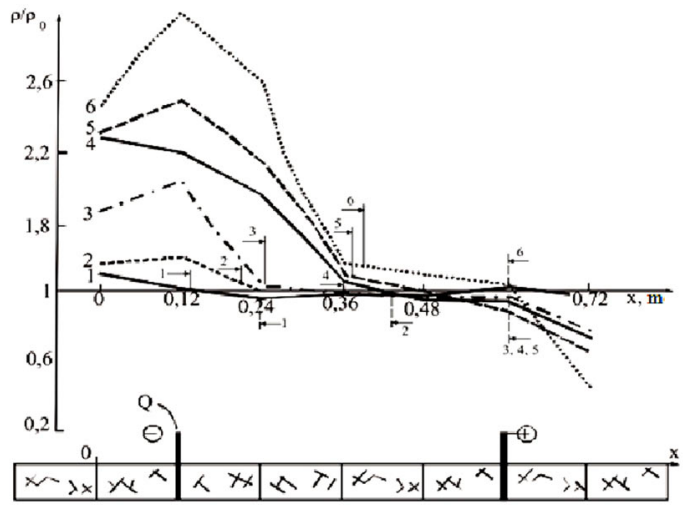

Fig. 2. Dependence of the relative SER $\rho / \rho_{0}$ on $I \cdot t$ and coordinates $x$ of the sensor installation in the electroosmotic treatment area: $1-I \cdot t=5.6 \mathrm{~A} \cdot \mathrm{h} ; 2-8 ; 3-18 ; 4-22 ; 5-30 ; 6-54$

$\longrightarrow$ the border of the drying zone; $\longleftarrow$ - the border of moisture accumulation area

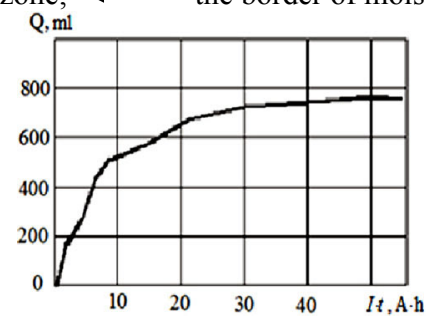

Fig. 3. The output of soil liquid $Q$

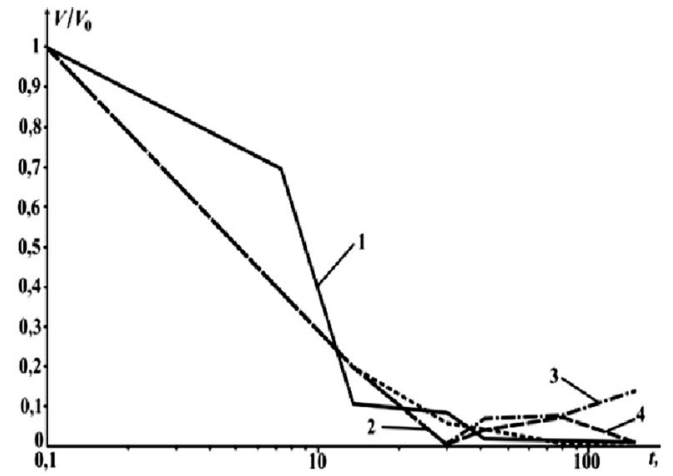

Fig. 4. Changes in the relative velocity of electroosmosis: 1 - the velocity calculated by the output of the liquid; 2, 3 and 4 - the calculated velocity in the drying zone, the intermediate zone and the zone of the accumulation of moisture, respectively.

Calculation of the electric field of the plant at the third stage is carried out using programs of section PDE of MATLAB libraries that implement finite element method, wherein the task in the heterogeneity model is implemented based on the following assumptions:

- the original massif had specific inductive capacitance $(\varepsilon=27)$ corresponding to the value taken for the wet clay;

- in the course of treatment, the above indicated characteristic zones are formed with value $\varepsilon$ corresponding to wet, moisturized and dry ( $\varepsilon=27,18$ and 7$)$ clay; 
- the dimensions of the computer model and the processing mode complied with the physical model, an example of which is given in the work [9], and the dimensions of the three zones varied in accordance with the data obtained at the first stage.

Fig. 5 shows the plots of changes in the electric field intensity $E$ along the main axis $\mathrm{x}$ of the unit in the transverse horizontal plane located at the middle section of the immersed electrode-injectors.

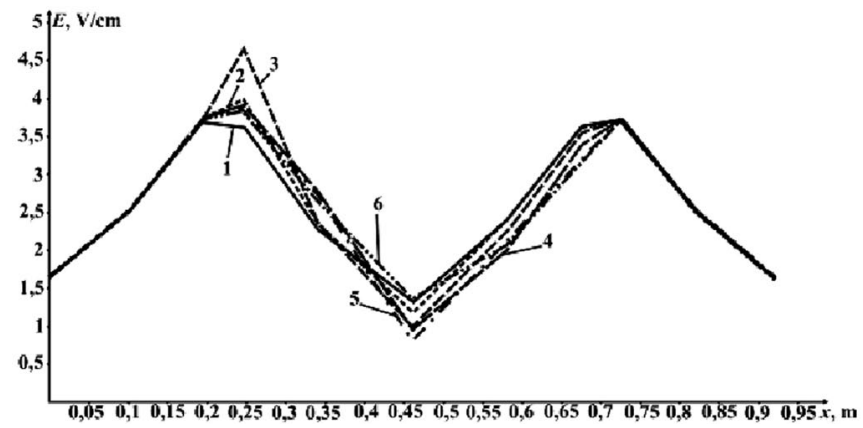

Fig. 5. Changing of the electric field intensity in the interelectrode space with time at depth $z=0.125$ m: 1 - 5.6 A.h; 2 - $8 \mathrm{~A} \cdot \mathrm{h} 3$ - $18 \mathrm{~A} \cdot \mathrm{h} ; 4$ - $22 \mathrm{~A} \cdot \mathrm{h} ; 5$ - $30 \mathrm{~A} \cdot \mathrm{h} ; 6$ - $54 \mathrm{~A} \cdot \mathrm{h}$

Having taken, in accordance with the experimental data, the initial value $k_{e 0}=1.3 \cdot 10^{-4}$ $\mathrm{cm}^{2} / \mathrm{V} \cdot \mathrm{s}$, and taking into account the average values of the relative filtration velocity by zones, we calculated the dependencies of electroosmotic and hydrodynamic parameters $k_{f}$, $k_{e}, k_{a}$ for each zone with time $t$ (Fig. 6) and depending on the coordinate $x$ (Fig. 7).

a

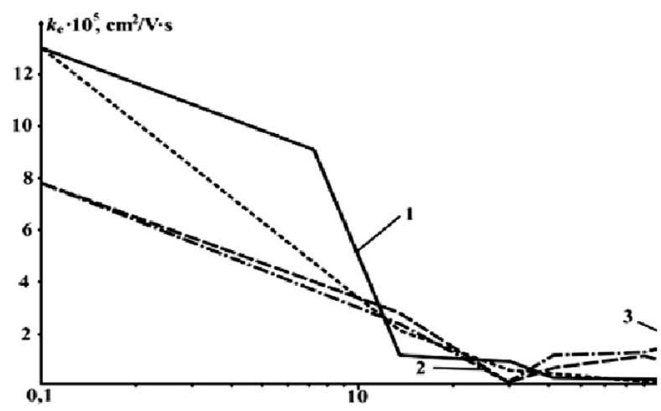

$\mathrm{b}$

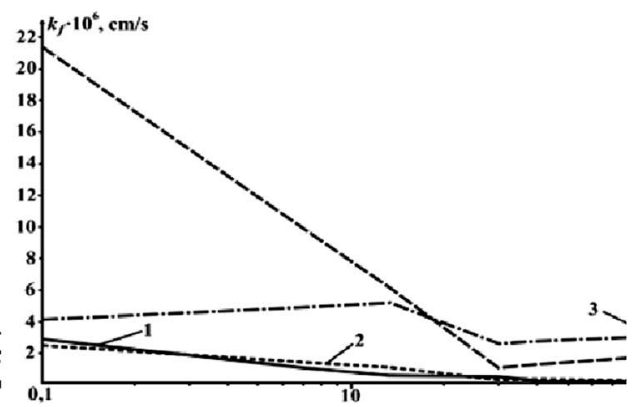

Fig. 6. Coefficients of electroosmotic permeability $k_{e}$ (a), hydraulic permeability $k_{f}$ (b) the versus time: 1 - calculation results for the output of liquid; 2 - drainage area; 3 - the transition zone; 4 moisture accumulation zone 

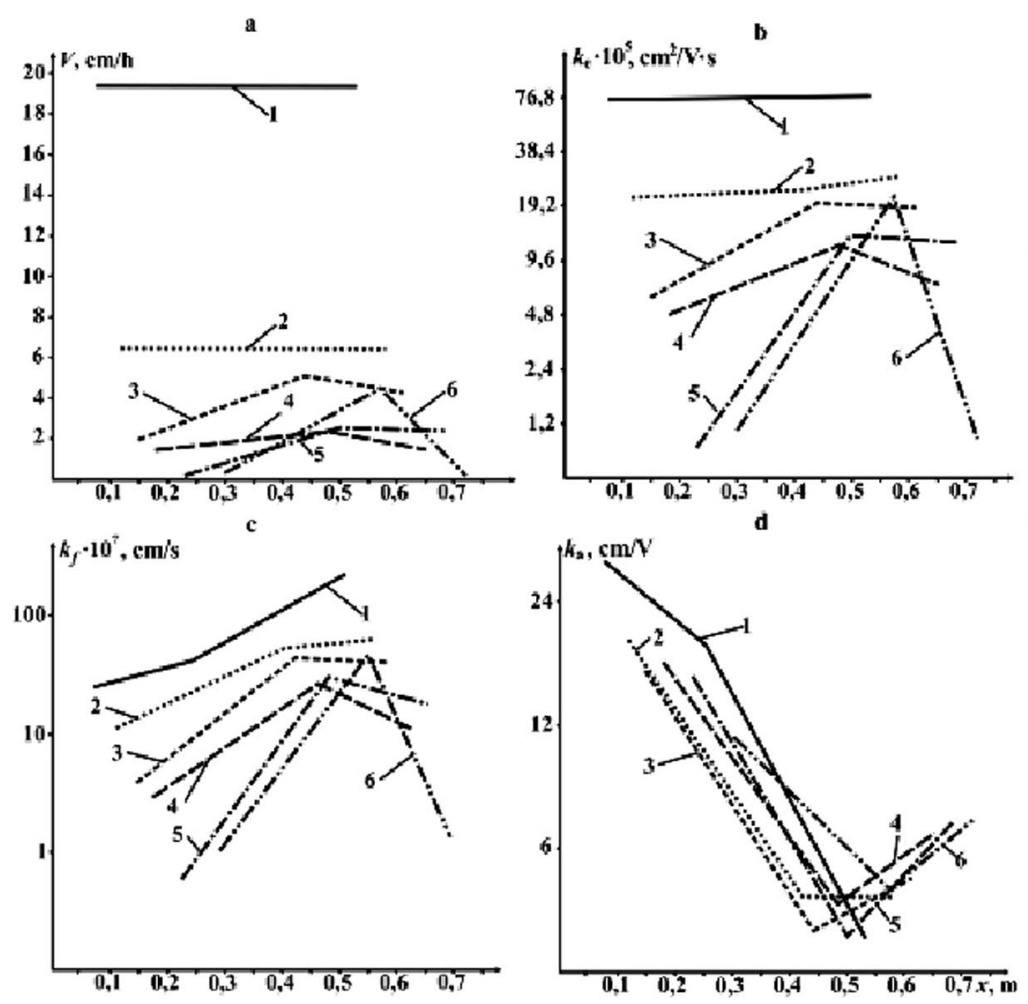

Fig. 7. Velocity of electroosmosis (a) and coefficients of electroosmotic permeability $k_{e}$ (b), hydraulic permeability $k_{f}$ (c) and the electroosmotic activity $k_{a}$ (d) versus the coordinate $\mathrm{x}$ along the length of the model: 1 - 5.6 A·h; 2 - $8 \mathrm{~A} \cdot \mathrm{h} 3$ - $18 \mathrm{~A} \cdot \mathrm{h} ; 4$ - $22 \mathrm{~A} \cdot \mathrm{h} ; 5$ - $30 \mathrm{~A} \cdot \mathrm{h} ; 6$ - $54 \mathrm{~A} \cdot \mathrm{h}$
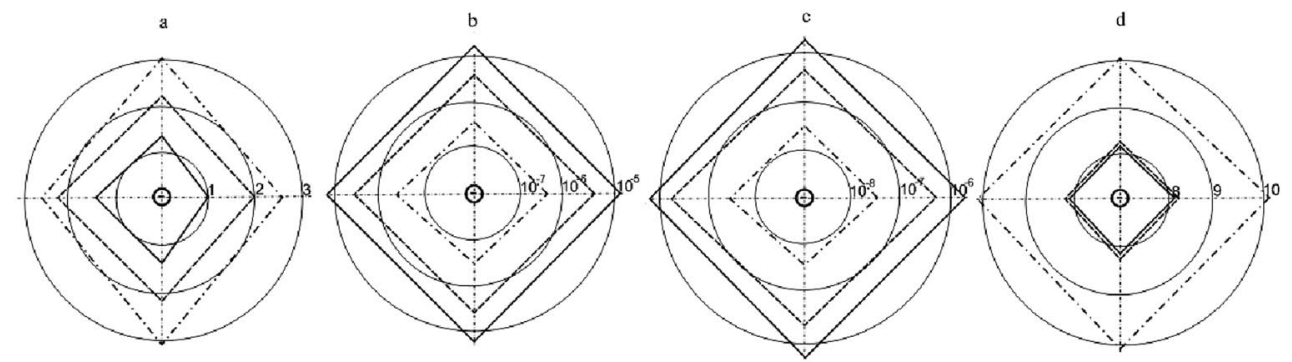

Fig. 8. The diagrams of change in the relative resistivity $\rho / \rho_{0}$ (a), $k_{e}, \mathrm{~cm}^{2} / \mathrm{V} \cdot \mathrm{s}(\mathrm{b}), k_{f}, \mathrm{~cm} / \mathrm{s}(\mathrm{c})$, $k_{a}, \mathrm{~cm} / \mathrm{V}(\mathrm{d}):--\mathrm{I} \cdot \mathrm{t}=8 \mathrm{~A} \cdot \mathrm{h} ; \quad--\cdot-\mathrm{I} \cdot \mathrm{t}=22 \mathrm{~A} \cdot \mathrm{h} ; \quad--\cdot \mathrm{f} \cdot \mathrm{t}=54 \mathrm{~A} \cdot \mathrm{h}$

\section{Conclusion}

The analysis of the results led to the following conclusions.

1. The developed method allows us to simulate unsteady hydrodynamic processes in the zone of soils subjected to electroosmotic treatment in the one-dimensional pattern, in the plane, and in space.

2. The differences in the ranges of values of electroosmotic permeability coefficient $k_{e}$ for the zones of humidification and dehumidification are negligible, but, in view of the results found by the analysis of the dynamic changes in the zones, these values are of the op- 
posite nature: in the course of drying the ke value decreases with time, and during moisture accumulation - it increases.

3 . The estimated values of the electroosmotic permeability coefficient $k_{e}$ with the decrease in the average value of electric field intensity in the range from 3.8 to $0.8 \mathrm{~V} / \mathrm{cm}$ also decrease in the range from $1.3 \cdot 10^{-4} \mathrm{~cm}^{2} / \mathrm{V} \cdot \mathrm{s}$ to $1.11 \cdot 10^{-6} \mathrm{~cm}^{2} / \mathrm{V} \cdot \mathrm{s}$.

4. The calculated values of hydraulic permeability coefficient $k_{f}$ in the range of $0.005-$ $0.02 \mathrm{~m} / \mathrm{d}$ correspond to the range of values from 0.005 to $0.05 \mathrm{~m} / \mathrm{d}$, in which using of the electroosmotic treatment of soils is more efficient than conventional methods of dewatering.

5. In the area of active electroosmosis in the process of electroosmotic filtration the depression is created with the movement of fluid to the electrode being relatively uniform in all the directions, as evidenced by symmetrical changes in the electrical and hydrodynamic parameters obtained by an experimental and analytical way (Figure 8, a), which is consistent with the nature of the dependencies presented in the study [18].

\section{References}

1. Khaled, The Application of Electroosmosis in Clay Improvement, 55 (2016)

2. Asadi, B. B. K. Huat, H. Nahazanan, H. A. Keykhah, Int. J. Electrochem. Sci., Theory of Electroosmosis in Soil, 8 (2013)

3. J.-L. Chen, L. Murdoch, ASCE, 5 (1999)

4. S. A. A. Tajudin, Electrokinetic stabilisation of soft clay (2012)

5. M. F. Buehler, J. E. Surma, J. W. Virden, Thirty-Third Hanford Symposium on Health, 36 (1994)

6. V. dos Santos, M. O. Medeiros, A. S. D. dos Anjos, C. A. Martínez-Huitle, D. R. da Silva, Chemical Engineering Transactions, 41, 6 (2014)

7. R.Z. Velten, D.C. Lima, M.P.F. Fontes, C.A.B. Carvalho, Soils and Rocks, 32, 11 (2012)

8. D. V. Morris, S. F. Hillis, J. A. Caldwell, Can. Geotech. J., 22, 8 (1985)

9. S. M. Prostov, E. A. Shabanov, The 8th Russian-Chinese Symposium. Coal in the 21st Century: Mining, Processing and Safety, 9 (2016)

10. Shao-Chi Chiena, Pio-Go Hsiehb, Chang-Yu Ou, ACEM 12, 11 (2012)

11. Y. Guo, Electrokinetic dewatering of oil sands tailings, 112 (2012)

12. F. Eriksson, L. Gemvik, Electro-Osmotic Treatment of Soil, 116 (2014)

13. S. Hansbo, Sixth International Conference on Case Histories in Geotechnical Engineering, 15 (2008)

14. S. M. Prostov, M. V. Gucal, E. A. Shabanov, Taishan Academic Forum, 8 (2014)

15. J. Yuan, M.A. Hicks, J. Dijkstra, ISSMGE - TC 211, 8 (2012)

16. Kit Design and modelling of electroosmotic dewatering, 2014 (2006)

17. S. R. Maduar, A. V. Belyaev, V. Lobaskin, O. I. Vinogradova, PRL, 114, 5 (2015)

18. Zhi-ming Liu, Jian-gui Yang, Qi-meng Li, EJGE, 21, 13 (2016) 